\title{
THE REVERSIBLE ADIABATIC EXPANSION OF WATER.
}

By J. R. Roebuck.

IF water be subjected to a change of pressure it is a matter of experiment that the temperature usually shifts slightly also. This temperature change depends upon the initial temperature, the initial pressure, and the pressure change. The work described here was undertaken on account of its bearing on the method for the determination of the mechanical equivalent of heat described in an earlier paper, ${ }^{1}$ where a quantitative knowledge in a limited region of the relation between these variables was required. This relation has been studied both experimentally and theoretically by G. Tammann ${ }^{2}$ who deduces the thermodynamic equation,

$$
\left(\frac{d t}{d p}\right)_{Q}=\frac{T}{C_{p}}\left(\frac{d v}{d t}\right)_{p}
$$

where $C_{p}$ is the specific heat of water at constant pressure and the subscript $Q$ indicates adiabatic conditions. He integrates this equation arithmetically and obtains a set of curves which will aid in the discussion of this work if they are reproduced here, Fig. I. The ordinates are temperature change from the initial temperature marked on each curve, and the

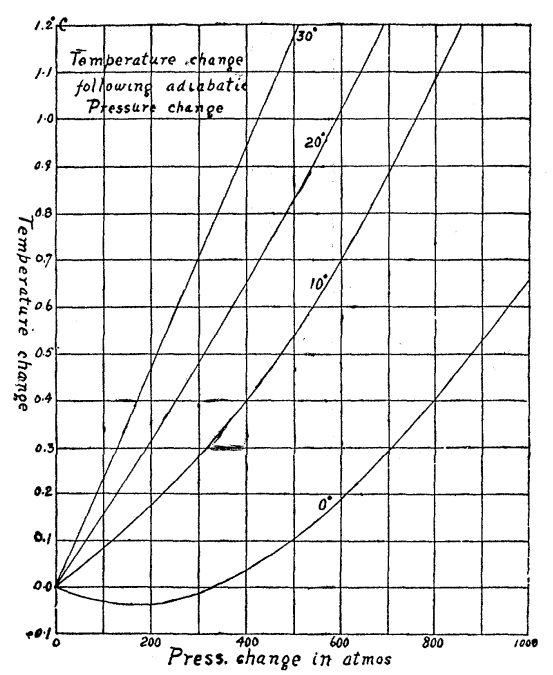

Fig. 1. final pressure is always the same, one atmosphere.

From these curves it is at once evident that by a suitable choice of temperature, pressure and pressure drop, the resulting change of temperature will be zero. It will also be evident that the pressure drop need not be always to one atmosphere, the condition in these curves, to have this condition filled. By the choice of one of these sets of conditions for

1 Phys. Rev., 2d Ser., 2, 79, r9r3.

2 Über die Beziehungen zwischen den inneren Kräften und Eigenschaften der Lösungen, p. I3I. 
the porous plug reading, the total temperature change occurring there (with a trifling correction) would be due to the work done on the water, that is, the only serious correction would be reduced to zero.

Except with two points of difference the apparatus used for the work on the mechanical equivalent of heat was used here. The first difference was that the higher pressures frequently used required other apparatus for its measurement than the open tube mercury manometer. Some experience with spring gauges has shown them to be quite unreliable for close work. The trouble seems unfortunately to be connected with the elastic properties of materials and less seriously with the multiplying

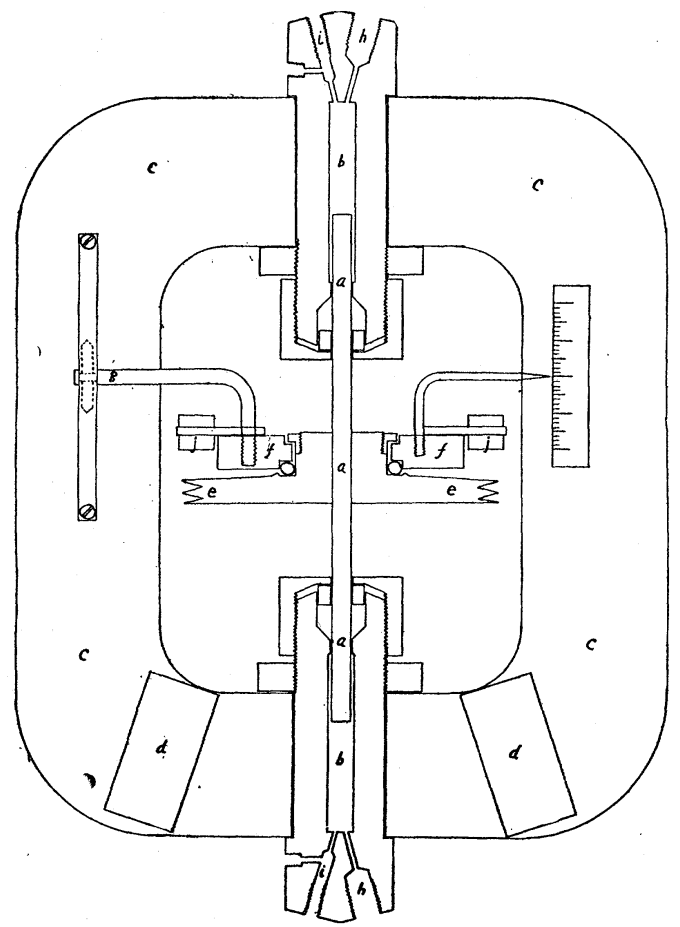

Fig. 2.

$a, a, a$, steel piston; $b, b$, cylinders; $c, c, c, c$, cast-iron yoke; $d, d$, lugs for legs; $e, e$, pulley; $f, f$, ring of ball bearing; $g$, arm for holding " $f$ " from rotating; $h, h$, unions for pressure leads; $i, i$, relief cocks; $j, j$, ring for gimbals.

mechanism. To summarize, the reading depends somewhat on the time during which the pressure is on the gauge, increasing slightly with time. The reading is materially different when approached from a higher pressure from that obtained when approached from a lower pressure and this quite beside any lost motion or friction in the recording mechanism. 
Physical Review, Vol. II., Second Series. October, I9I3.

Plate I.

To face page $30 r$.

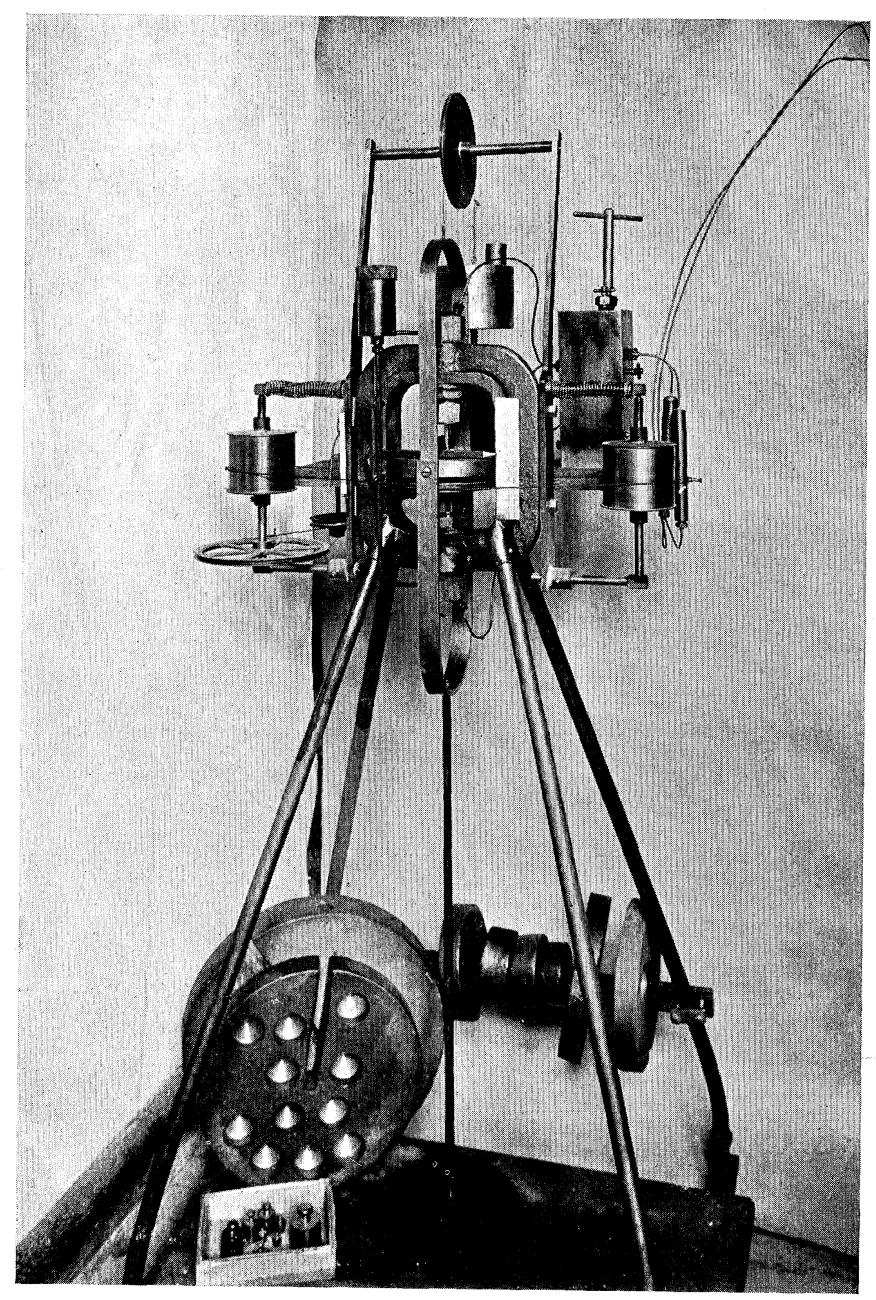

Fig. 3.

J. R. ROEBUCK. 
The elasticity of the spring tube depends upon the temperature. Beside these grave faults, many even of the expensive gauges have serious errors due to the multiplying mechanism such as lost motion, friction and irregularity due to gear transmission. Their great advantages are their rapidity and ease of reading.

Experience with the rotating-piston pressure regulator had proved it to be so reliable, that a modified type of the engineer's dead-weight tester promised to give satisfactory results. Accordingly a differential manometer of this type was designed and its use in this and other projected work justifies a full description. It was built in the department shop by Mr. Foerst and no small part of its excellent performance was due to his accurate and careful work. A vertical section is shown in Fig. 2 and a photograph of the complete instrument in Fig. 3. Two cylinders (b) face each other on a common axis in a cast-iron frame $(c)$ and are entered through leather packed stuffing boxes by a common piston $(a)$ of glasshard steel carefully ground and polished. At its center this piston carries a double grooved pulley $(e)$ for rotating by round belts from two opposite cylinders under nearly equal tension. On the upper side of this grooved pulley $(e)$ is a thrust ball-bearing carrying through gimbals ( $f$ and $j$ ) and a steel $\mathrm{U}$, a vertical iron rod with a disk at the lower end for supporting the load of slotted iron weights. The arm $(g)$ holds the gimbals from rotating while it allows vertical motion. All of these parts except the load are counterpoised by a weight hung over a pulley supported on hard-steel knife-edges. An iron cylinder attached to this counterpoise dips in mercury and serves to give the rotating cylinder a definite vertical equilibrium position. It is provided with a scale and was calibrated for displacement by small known weights. The cylinders were filled with paraffin oil and connected to the water system through U-tubes where the oil floated on the water. A block of steel carried the necessary relief cocks and valves. A single pressure was read by balancing the pressure admitted only to the lower cylinder, by a load of the iron weights; while a difference of pressure was read by admitting the higher pressure to the lower cylinder, the lower pressure to the upper cylinder, and balancing the difference by the load. The pistons were so nearly the same size that when in the symmetrical equilibrium position the same pressure up to 300 atmospheres applied simultaneously to the two pistons did not displace the zero beyond the experimental error of setting. The piston always returned from either direction in three to five minutes to within a $\mathrm{mm}$. of the equilibrium position. A displacement of a mm. corresponded to $0.0 \mathrm{ll}$ lb. load, or $0.2 \mathrm{lb}$. per sq. in. pressure. The slotted iron weights were standardized against the state standards at 
the state laboratory, for which courtesy my thanks are due to Mr. Dowling, in charge there. The diameter of the piston was measured with a micrometer calipers and over the part in use was exceedingly true. A simultaneous reading of a pressure on this instrument and on the open tube mercury manometer checked with this measurement of the diameter to $0 . \mathrm{I}$ per cent. There was no leak past the leather packing and under these conditions it is difficult to see how a compression of the steel piston by the pressure used could influence the effective area of cross-section. Measurements of pressure due to the rotating-piston regulator could be duplicated to o.I per cent.

The second difference in the experimental arrangement was in the plug chamber. The plug was removed and the thermometer which had been inside the plug served for these readings. The other thermometer was removed to the bath and its place in the pressure system was taken by a device for stirring the water in the plug chamber immediately about the thermometer. A cylinder of very thin brass was placed around the thermometer and stirrer, so that the stirred water whose temperature changes were followed, was surrounded by a cylinder of water, not stirred, but subjected to the same pressure vibrations. The stirring of the inner chamber had to be limited to a very gentle movement once per minute or the effect of it showed at once on the thermometer.

In reading platinum resistance thermometers the best procedure is to use a continuous current so that the heating of the thermometer coils will be similar. In this work the addition of this energy to the water about the thermometer would have led to a marked rise of temperature when continued over the time for which the experiment lasted, so that it became necessary to make the bridge settings by momentary contacts of the Callendar key. This involves reading a small induction effect as resistance, though the bridge coils and thermometers were wound noninductively, but since the resistance was varying within very narrow limits, a few thousandths of a degree at most, the induction effect would go out in the process of subtraction of the resistances to get the temperature change.

The experimental procedure was as follows: After the thermostat had been in steady operation for at least an hour, the stirrer was operated and the temperature read to $0.000 \mathrm{I}^{\circ} \mathrm{C}$., once per minute, till the temperature became constant to about this amount, when the pressure was changed suddenly the predetermined amount by changing the load on the regulator, and the temperature again followed for about ten minutes. Usually in four to six minutes it was constant again, and in case the temperature change was large it often showed a drift at the end of the 
ten minutes. After an interval of thirty minutes to one hour this experiment was followed by a duplicate determination except that the pressure change had the opposite sign. The two readings usually checked to within $0.0003^{\circ} \mathrm{C}$. but some showed large differences due probably to thermostat irregularities.

The temperature changes immediately following the pressure change are of some interest. Fig. 4 shows a typical curve, for a relief of pressure. Immediately following the pressure drop the thermometer records a sudden fall of tem-

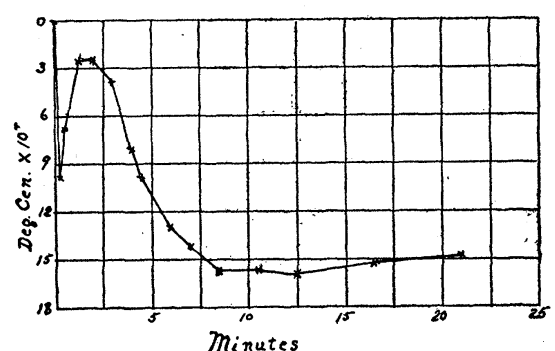

Fig. 4. perature due probably to the work done by the thermometer case against the falling pressure. This is followed by a rapid return to the temperature of the water which may be above or below the initial temperature. In the case figured the temperature fell $0.0016^{\circ} \mathrm{C}$.

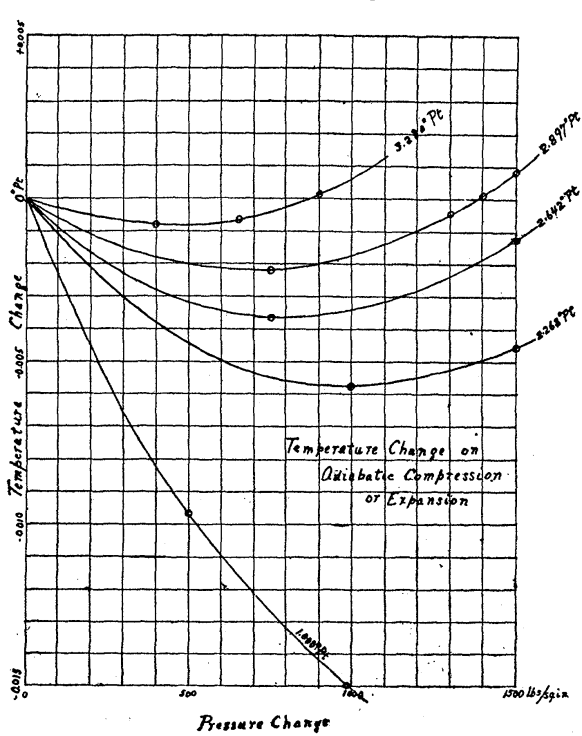

Fig. 5.

To explore the field generally a limited set of readings was taken between $0^{\circ}$ and $4^{\circ} \mathrm{C}$., with the lower pressure always one atmosphere, and the results are plotted in Fig. 5. This method of building up the curves does not give those which are the most useful. For each curve the initial temperature and final pressure are always the same, so that the curve does not represent exactly the path of the water during an expansion. For this either both initial or both final conditions should be alike for all points on a curve. But since the temperature change is so small and such a slight function of the initial temperature, the difference between the methods is immaterial. The curves are of the same form as Tammann's in Fig. I. On attempting a numerical check it was soon apparent that the data on the coefficient of expansion at these temperatures were so uncertain as to make the calculations of little value. In the case of one reading at $26^{\circ} \mathrm{C}$. the agreement was within 
the accuracy of reading from the original figure given by Tammann. Certain of these curves cut the axis for zero temperature change, which were the points of particular interest for this work. In general the temperature change is a function of the initial temperature, the initial pressure, and the pressure change. The particular value, $\Delta t=0$, is con-

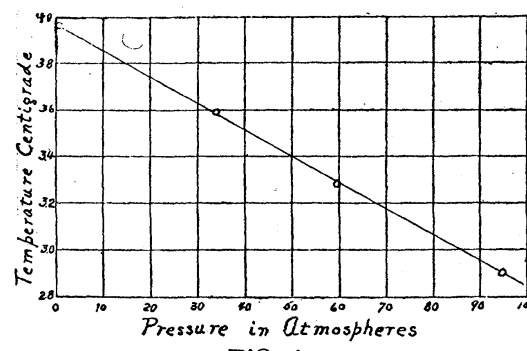

Fi.8. 6. sequently a function of all three variables. Fig. 6 gives the $(p, t)$ relation of these points for the final pressure always one atmosphere. The curve cuts the temperature axis close to $3.96^{\circ} \mathrm{C}$., in good agreement with the temperature of maximum density of water at atmospheric pressure, as was to be expected from Tammann's equation. The point at $3.59^{\circ} \mathrm{C}$. was determined with especial care by varying the temperature at which the reading was made using the same initial and final pressures, and the readings are plotted in Fig. 7. This curve and the corresponding curve estimated from Fig. 5

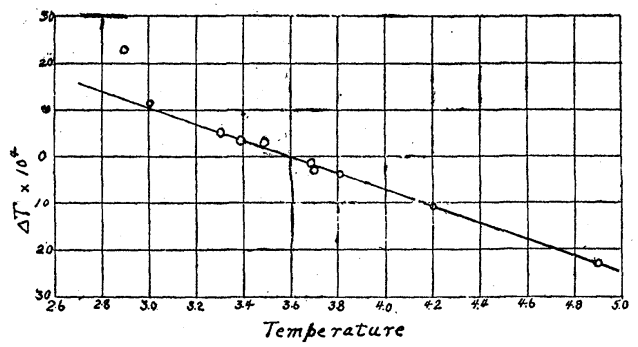

Fig. 7.

give the temperature and pressure limits between which the mechanical equivalent had to be determined with this final pressure so that the correction should fall below $0.000 \mathrm{I}^{\circ} \mathrm{C}$.

From Tammann's equation given above it is at once evident that when $(d t / d p)_{q}=o$, then also $(d v / d t)_{q}=0$, since the other quantities involved do not go to zero. That is to say, the maximum density conditions are also conditions where the adiabatic rate of change of temperature with change of pressure is zero. This offered another chance for experimental test of the theory and it was hoped might also prove to be a satisfactory method for the determination of the pressure-temperature relations of the maximum density. But the method proved exceedingly laborious and the results did not justify the labor required. The desired data called for the determination of $\Delta t / \Delta p$ at the limit $\Delta p=0$. As a rule this 
is not possible and approximations have to suffice. Here it is possible, since the rate has positive, zero, and negative values. The procedure is to find a sufficient group of sets of conditions under each set of which $\Delta t=0$; from which it is possible to interpolate for the set of conditions under which $\Delta p=0$. The experiments deal with four variables and following the usual plan two are kept constant while the relation between the other two is being determined. The two, $p$ and $\Delta t$, were chosen as being the most readily managed with the experimental set-up. A series of readings were made at constant temperature, of the temperature change due to a constant pressure drop from a series of initial pressures. If all four variables were chosen suitably, the curve obtained by plotting the experimental values of $p$ and $\Delta t$ cut the axis for $p$ at which point $\Delta t=0$. It was only necessary to plot the values as the readings progressed when from the trend of the curve $p$ could be so chosen as to make $\Delta t$ go through the zero value. This was repeated for other values of $\Delta p$ but the same temperature, each curve giving a value of $p$ and $\Delta p$ at which for this temperature $\Delta t=0$. Plotting of these values of $p$ and $\Delta p$ gave a curve cutting the $p$ axis where $\Delta p=0$, or at least by indicating the trend of the curve enabled the experiments to be so chosen as to make the curve cut this axis. For this cutting point then $\Delta p=0$, also $\Delta t=0$, so that $(d t / d p)_{\boldsymbol{q}}=0$, and the values of $p$ and $t$ therefore describe maximum density conditions. The whole process had then to be repeated for other constant temperatures, each repetition giving a new point on the maximum density pressure-temperature curve. A large number of

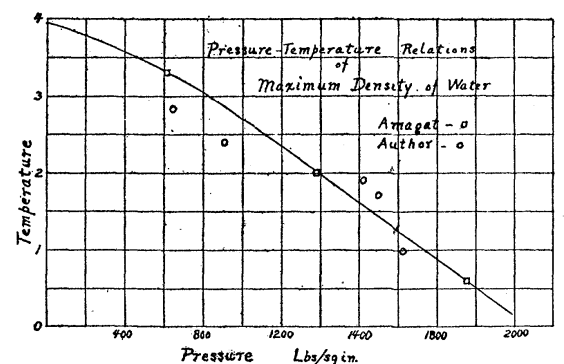

Fig. 8.

readings were required to fix one of these points. The determination in duplicate of four to six points required a day's work. The data as reported could be improved by a larger number of readings, but the results did not promise to be as reliable as those by direct measurement of volume. None of the actual data is reported but only the final curve, Fig. 8. The points fall around Amagat's curve for the maximum density and hence may be taken as supporting Tammann's equation. 
The principal contents of this paper are:

I. Description of an accurate rotating-piston differential manometer.

2. The method and data for fixing the point at $3.59^{\circ} \mathrm{C}$. for the method for the determination of the mechanical equivalent of heat.

3. Experimental test of Tammann's curves, both direct and indirect.

4. Describes, tests and rejects an indirect method for the determination of the $(p, t)$ relations of the maximum density of water.

Department of Physics,

UNIVERSITY OF WISCONSIN,

June, I9r3. 


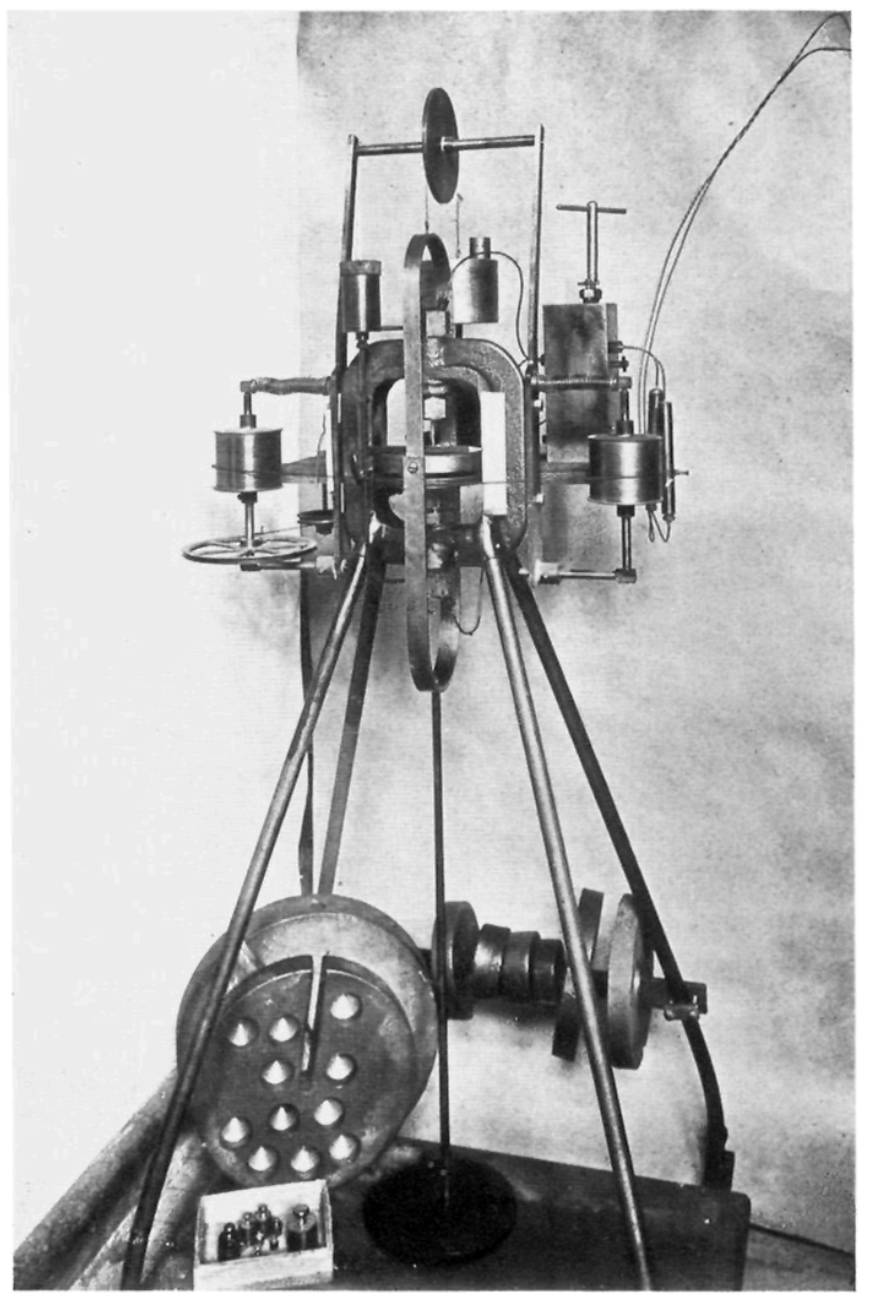

Fig. 3. 